PROCEEDINGS OF THE

AMERICAN MATHEMATICAL SOCIETY

Volume 133, Number 1, Pages 223-228

S 0002-9939(04)07602-6

Article electronically published on July 26, 2004

\title{
EXISTENCE AND UNIQUENESS OF SOLUTIONS FOR QUASILINEAR ELLIPTIC SYSTEMS
}

\author{
D. D. HAI
}

(Communicated by David S. Tartakoff)

\begin{abstract}
We obtain necessary and sufficient conditions for the existence of positive solutions for a class of sublinear Dirichlet quasilinear elliptic systems.
\end{abstract}

\section{INTRODUCTION}

Consider the quasilinear elliptic system

$$
\left\{\begin{array}{cl}
-\Delta_{p} u=\lambda f(v) & \text { in } \Omega, \\
-\Delta_{q} v=\mu g(u) & \text { in } \Omega, \\
u=v=0 & \text { on } \partial \Omega,
\end{array}\right.
$$

where $f, g:[0, \infty) \rightarrow[0, \infty), \Omega$ is a bounded domain in $R^{N}$ with smooth boundary $\partial \Omega, \Delta_{p} u=\operatorname{div}\left(|\nabla u|^{p-2} \nabla u\right), \Delta_{q} v=\operatorname{div}\left(|\nabla v|^{q-2} \nabla v\right), p, q>1$, and $\lambda, \mu$ are positive parameters.

Recently, Dalmasso 2 studied existence and uniqueness of positive solutions to (I) when $p=q=2$ and $f(c g(x))$ is sublinear at 0 and $\infty$ for every $c>0$. Related results in the case when $f(0)<0$ or $g(0)<0$ are obtained in [4. In this paper, we are interested in the existence and uniqueness of positive solutions for the quasilinear system (I) when $f^{1 /(p-1)}\left(c g^{1 /(q-1)}(x)\right)$ is sublinear at 0 and $\infty$ for every $c>0$. We also show under additional assumptions that these conditions are necessary for (I) to have a positive solution for all $\lambda, \mu>0$. Our results complement and extend corresponding results in [2]. Our approach depends on fixed points arguments and maximum principles.

\section{MAIN RESULTS}

We make the following assumptions:

(H.1) $f, g:[0, \infty) \rightarrow[0, \infty)$ are continuous, nondecreasing, and $g(x)>0$ for $x>$ 0 .

(H.2) For each $c>0$,

$$
\limsup _{x \rightarrow 0^{+}} \frac{f^{\frac{1}{p-1}}\left(c g^{\frac{1}{q-1}}(x)\right)}{x}=\infty .
$$

Received by the editors September 30, 2003.

2000 Mathematics Subject Classification. Primary 35J25, 35J70.

Key words and phrases. Uniqueness, quasilinear elliptic systems, positive solutions.

(C)2004 American Mathematical Society 
(H.3) For each $c>0$,

$$
\liminf _{x \rightarrow \infty} \frac{f^{\frac{1}{p-1}}\left(c g^{\frac{1}{q-1}}(x)\right)}{x}=0 .
$$

Then we have

Theorem 1. Let (H.1)-(H.3) hold. Then (I) has a positive solution $(u, v)$ for all $\lambda, \mu>0$.

Theorem 2. Let $f, g$ satisfy (H.1). Suppose that there exist positive numbers $r, s$ with $r s<(p-1)(q-1)$ such that

$$
\frac{f(x)}{x^{r}} \text { and } \frac{g(x)}{x^{s}}
$$

are nonincreasing for $x \geq 0$. Then (I) has at most one positive solution.

Theorem 3. Let (H.1) hold, and suppose that there exist positive numbers $k_{1}, k_{2}$ such that

$$
\liminf _{x \rightarrow 0^{+}} \frac{f^{\frac{1}{p-1}}\left(k_{1} g^{\frac{1}{q-1}}(x)\right)}{x}>0
$$

and

$$
\limsup _{x \rightarrow \infty} \frac{f^{\frac{1}{p-1}}\left(k_{2} g^{\frac{1}{q-1}}(x)\right)}{x}<\infty .
$$

Suppose that (I) has a positive solution for all $\lambda, \mu>0$. Then for each $c>0$,

$$
\limsup _{x \rightarrow 0^{+}} \frac{f^{\frac{1}{p-1}}\left(c g^{\frac{1}{q-1}}(x)\right)}{x}=\infty
$$

and

$$
\liminf _{x \rightarrow \infty} \frac{f^{\frac{1}{p-1}}\left(c g^{\frac{1}{q-1}}(x)\right)}{x}=0 .
$$

Remarks. 1. Theorems 1 and 2 were established in [2] in the case when $p=q=2$.

2. The conclusion of Theorem 3 holds under the weaker assumption that (I) has a positive solution for $\lambda, \mu$ small and $\lambda, \mu$ large.

3. Let $f(x)=x^{r}+1, g(x)=x^{s}$, where $r, s>0$ and $r s<(p-1)(q-1)$. Then $f, g$ satisfy the assumptions of Theorems 1 and 2, thus giving the existence and uniqueness of a positive solution of (I).

4. Let $f(x)=e^{\alpha x}, g(x)=(\ln (1+x))^{\beta}$ where $\alpha, \beta>0$ and $\beta \leq q-1$. Then $(*)$ is satisfied for all $k_{1}>0$ and $(* *)$ is satisfied for small $k_{2}>0$. It follows from Theorems 1 and 3 that (I) has a positive solution for all $\lambda, \mu>0$ if and only if $\beta<q-1$.

Let $\phi, \psi$ satisfy

$$
\begin{aligned}
& -\Delta_{p} \phi=1 \text { in } \Omega, \phi=0 \text { on } \partial \Omega, \\
& -\Delta_{q} \psi=1 \text { in } \Omega, \psi=0 \text { on } \partial \Omega .
\end{aligned}
$$

Let $D$ be a sub-domain of $\Omega$ with $\bar{D} \subset \Omega$. Let

$$
h(x)=\left\{\begin{array}{l}
1 \text { if } x \in D, \\
0 \text { if } x \notin D,
\end{array}\right.
$$


and let $\tilde{\phi}, \tilde{\psi}$ be the solution of

$$
\left\{\begin{aligned}
-\Delta_{p} \tilde{\phi} & =h \quad \text { in } \Omega \\
\tilde{\phi} & =0 \text { on } \partial \Omega
\end{aligned}\right.
$$

and

$$
\left\{\begin{aligned}
-\Delta_{q} \tilde{\psi}=h & \text { in } \Omega, \\
\tilde{\psi}=0 & \text { on } \partial \Omega,
\end{aligned}\right.
$$

respectively. By the strong maximum principle (see [7]), there exist positive numbers $M, m$ such that $\tilde{\phi} \geq M \phi$ in $\Omega$ and $\phi, \psi, \tilde{\phi}, \tilde{\psi} \geq m$ in $\bar{D}$.

Without loss of generality, we assume that $\lambda=\mu=1$ in the proof of Theorems 1 and 2.

Proof of Theorem 1. By (H.2), there exists $\varepsilon \in(0,1)$ such that

$$
M f^{\frac{1}{p-1}}\left(m g^{\frac{1}{q-1}}(\varepsilon m)\right) \geq \varepsilon .
$$

For each $w \in C(\bar{\Omega})$, let $u=T w$ be the solution of

$$
\begin{cases}-\Delta_{p} u=f(v) & \text { in } \Omega, \\ -\Delta_{q} v=g(\max (w, \varepsilon \phi)) & \text { in } \Omega, \\ u=v=0 & \text { on } \partial \Omega .\end{cases}
$$

Then $T: C(\bar{\Omega}) \rightarrow C(\bar{\Omega})$ is completely continuous (see e.g. [3], [5]). By (H.3), there exists a number $R>|\phi|_{\infty}$ such that

$$
f^{\frac{1}{p-1}}\left(|\psi|_{\infty} g^{\frac{1}{q-1}}(R)\right)|\phi|_{\infty} \leq R .
$$

We claim that $T: \bar{B}(0, R) \rightarrow \bar{B}(0, R)$, where $\bar{B}(0, R)$ denotes the closed ball centered at 0 with radius $R$ in $C(\bar{\Omega})$. Indeed, let $w \in C(\bar{\Omega})$ with $|w|_{\infty} \leq R$. Then we have

$$
-\Delta_{q} v=g(\max (w, \varepsilon \phi)) \leq g(R) \text { in } \Omega,
$$

which implies by the maximum principle that

$$
v \leq g^{\frac{1}{q-1}}(R) \psi .
$$

Thus

$$
-\Delta_{p} u=f(v) \leq f\left(g^{\frac{1}{q-1}}(R) \psi\right) \leq f\left(|\psi|_{\infty} g^{\frac{1}{q-1}}(R)\right),
$$

and therefore

$$
u \leq f^{\frac{1}{p-1}}\left(|\psi|_{\infty} g^{\frac{1}{q-1}}(R)\right) \phi .
$$

Consequently,

$$
|u|_{\infty} \leq f^{\frac{1}{p-1}}\left(|\psi|_{\infty} g^{\frac{1}{q-1}}(R)\right)|\phi|_{\infty} \leq R,
$$

proving the claim.

By the Schauder fixed point theorem, $T$ has a fixed point $u$ with $|u|_{\infty} \leq R$. Next, we verify that $u \geq \varepsilon \phi$. Since

$$
-\Delta_{q} v=g(\max (u, \varepsilon \phi)) \geq \begin{cases}g(\varepsilon m) & \text { in } D, \\ 0 & \text { in } \Omega \mid D,\end{cases}
$$

it follows from the maximum principle that

$$
v \geq g^{\frac{1}{q-1}}(\varepsilon m) \tilde{\psi} .
$$


Using this in the equation for $u$ gives

$$
-\Delta_{p} u=f(v) \geq \begin{cases}f\left(m g^{\frac{1}{q-1}}(\varepsilon m)\right) & \text { in } D, \\ 0 & \text { in } \Omega \mid D,\end{cases}
$$

and therefore

$$
u \geq f^{\frac{1}{p-1}}\left(m g^{\frac{1}{q-1}}(\varepsilon m)\right) \tilde{\phi} \geq M f^{\frac{1}{p-1}}\left(m g^{\frac{1}{q-1}}(\varepsilon m)\right) \phi \geq \varepsilon \phi .
$$

Since $g(x)>0$ for $x>0$, we have $v>0$ in $\Omega$ by the strong maximum principle. This completes the proof of Theorem 1.

Proof of Theorem 2. Let $(u, v)$ and $\left(u_{1}, v_{1}\right)$ be positive solutions of (I). As in [1], [6], we define $\delta=\sup \left\{\varepsilon>0: v \geq \varepsilon v_{1}\right.$ in $\left.\Omega\right\}$. Then $v \geq \delta v_{1}$. If $\delta<1$, then we have

$$
-\Delta_{p} u=f(v) \geq f\left(\delta v_{1}\right) \geq \delta^{r} f\left(v_{1}\right) .
$$

Since

it follows that

$$
-\Delta_{p}\left(\delta^{\frac{r}{p-1}} u_{1}\right)=\delta^{r} f\left(v_{1}\right)
$$

Using this in the equation for $v$ gives

$$
u \geq \delta^{\frac{r}{p-1}} u_{1}
$$

$$
-\Delta_{q} v=g(u) \geq g\left(\delta^{\frac{r}{p-1}} u_{1}\right) \geq \delta^{\frac{r s}{p-1}} g\left(u_{1}\right),
$$

which implies

$$
v \geq \delta \frac{r s}{(p-1)(q-1)} v_{1}
$$

a contradiction with the definition of $\delta$. Thus $\delta=1$, i.e., $v \geq v_{1}$, and so $v=v_{1}, u=$ $u_{1}$, proving Theorem 2 .

Before proving Theorem 3, we first establish

Lemma 4. Suppose that (H.1) and $\left(^{*}\right)$ hold, and let $\sigma, \eta>0$ be such that

$$
f^{\frac{1}{p-1}}\left(k_{1} g^{\frac{1}{q-1}}(x)\right) \geq \sigma x \quad \text { for } x \in[0, \eta] .
$$

Then there exists a positive number $K>0$ such that for $\lambda, \mu>K$, any positive solution $(u, v)$ of (I) satisfies

where $C=\frac{\eta}{|\phi|_{\infty}}$.

$$
u \geq C \phi
$$

Proof. Let $(u, v)$ be a positive solution of (I), and let $\alpha=\sup \{\varepsilon>0: u \geq \varepsilon \phi$ in $\Omega$. Then $\alpha>0$ and $u \geq \alpha \phi$. We claim that $\alpha \geq C$ if $\lambda, \mu$ are sufficiently large. Suppose to the contrary that $\alpha<C$. We have

$$
-\Delta_{q} v=\mu g(u) \geq \mu g(\alpha \phi) \geq \begin{cases}\mu g(\alpha m) & \text { in } D \\ 0 & \text { in } \Omega \mid D,\end{cases}
$$

and so

This, in turn, implies

$$
v \geq(\mu g(\alpha m))^{\frac{1}{q-1}} \tilde{\psi}
$$

$$
-\Delta_{p} u=\lambda f(v) \geq \lambda f\left((\mu g(\alpha m))^{\frac{1}{q-1}} \tilde{\psi}\right)
$$

and

$$
u \geq \lambda^{\frac{1}{p-1}} f^{\frac{1}{p-1}}\left((\mu g(\alpha m))^{\frac{1}{q-1}} m\right) \tilde{\phi} \geq \lambda^{\frac{1}{p-1}} f^{\frac{1}{p-1}}\left((\mu g(\alpha m))^{\frac{1}{q-1}} m\right) M \phi
$$


follows. If $m \mu^{1 /(q-1)}>k_{1}$ and $\lambda^{\frac{1}{p-1}} \sigma M m>2$, then since $\alpha m<C|\phi|_{\infty}=\eta$, it follows from (3) and (4) that

$$
u \geq \lambda^{\frac{1}{p-1}} f^{\frac{1}{p-1}}\left(k_{1} g^{\frac{1}{q-1}}(\alpha m)\right) M \phi \geq \lambda^{\frac{1}{p-1}} \sigma \alpha m M \phi>2 \alpha \phi,
$$

a contradiction with the definition of $\alpha$. This completes the proof of Lemma 4 .

Proof of Theorem 3. Let $c>0$, and suppose that (I) has a positive solution $(u, v)$ for every $\lambda, \mu>0$. Then we have

$$
|u|_{\infty} \leq\left(\lambda f\left(|v|_{\infty}\right)\right)^{\frac{1}{p-1}}|\phi|_{\infty}
$$

and

$$
|v|_{\infty} \leq\left(\mu g\left(|u|_{\infty}\right)\right)^{\frac{1}{q-1}}|\psi|_{\infty}
$$

Hence

$$
|u|_{\infty} \leq \lambda^{\frac{1}{p-1}} f^{\frac{1}{p-1}}\left(\mu^{\frac{1}{p-1}}|\psi|_{\infty} g^{\frac{1}{q-1}}\left(|u|_{\infty}\right)\right)|\phi|_{\infty} .
$$

For $\mu^{\frac{1}{p-1}}|\psi|_{\infty}<c_{0} \equiv \min \left(c, k_{2}\right)$, this implies

$$
\frac{f^{\frac{1}{p-1}}\left(c_{0} g^{\frac{1}{q-1}}\left(|u|_{\infty}\right)\right)}{|u|_{\infty}} \geq \frac{f^{\frac{1}{p-1}}\left(\mu^{\frac{1}{p-1}}|\psi|_{\infty} g^{\frac{1}{q-1}}\left(|u|_{\infty}\right)\right)}{|u|_{\infty}} \geq \frac{1}{\lambda^{\frac{1}{p-1}}|\phi|_{\infty}} .
$$

Using (**) and (5), we deduce that $\left|u_{\lambda}\right|_{\infty} \equiv|u|_{\infty}$ is bounded for $\lambda$ small, and hence

$$
\lim _{\lambda \rightarrow 0^{+}}\left|u_{\lambda}\right|_{\infty}=0
$$

Combining (5) and (6) gives

$$
\limsup _{x \rightarrow 0^{+}} \frac{f^{\frac{1}{p-1}}\left(c g^{\frac{1}{q-1}}(x)\right)}{x}=\infty .
$$

Next, let $\lambda, \mu$ be large enough so that $\lambda, \mu>K, \lambda^{\frac{1}{p-1}} m \min \left(a_{1}, k\right)>1$, and $m \mu^{\frac{1}{q-1}}>\max (1, c)$, where

$$
\begin{aligned}
a_{1} & =f^{\frac{1}{p-1}}\left(g^{\frac{1}{q-1}}(C m)\right), \\
k & =f^{\frac{1}{p-1}}\left(g^{\frac{1}{q-1}}(1)\right) .
\end{aligned}
$$

By Lemma 4,

$$
-\Delta_{q} v=\mu g(u) \geq \mu g(C \phi)
$$

and, using the arguments as in the proof of Lemma 4, we obtain

$$
v \geq \mu^{\frac{1}{q-1}} g^{\frac{1}{q-1}}(C m) \tilde{\psi} .
$$

Similarly, using (7) in the equation for $u$ gives

$$
u \geq \lambda^{\frac{1}{p-1}} f^{\frac{1}{p-1}}\left(\mu^{\frac{1}{q-1}} m g^{\frac{1}{q-1}}(C m)\right) \tilde{\phi} \geq \lambda^{\frac{1}{p-1}} a_{1} \tilde{\phi} .
$$

By repeating the arguments, we obtain a sequence of positive numbers $\left(a_{n}\right)$ such that for $n=1,2, \ldots$,

$$
\begin{aligned}
u & \geq \lambda^{\frac{1}{p-1}} a_{n} \tilde{\phi} \\
a_{n+1} & =f^{\frac{1}{p-1}}\left(m \mu^{\frac{1}{q-1}} g^{\frac{1}{q-1}}\left(\lambda^{\frac{1}{p-1}} m a_{n}\right)\right) .
\end{aligned}
$$


Clearly, $\left(a_{n}\right)$ is monotone and bounded, thus converging to a limit $a_{\lambda}$. An induction argument shows that

$$
a_{n} \geq k \quad \text { for } n \geq 2 \text {. }
$$

Hence $a_{\lambda} \geq k>0$ and

$$
a_{\lambda}=f^{\frac{1}{p-1}}\left(m \mu^{\frac{1}{q-1}} g^{\frac{1}{q-1}}\left(\lambda^{\frac{1}{p-1}} m a_{\lambda}\right)\right) .
$$

This implies

$$
\frac{f^{\frac{1}{p-1}}\left(c g^{\frac{1}{q-1}}\left(z_{\lambda}\right)\right)}{z_{\lambda}} \leq \frac{f^{\frac{1}{p-1}}\left(m \mu^{\frac{1}{q^{q-1}}} g^{\frac{1}{q-1}}\left(\lambda^{\frac{1}{p-1}} m a_{\lambda}\right)\right)}{\lambda^{\frac{1}{p-1}} m a_{\lambda}}=\frac{1}{\lambda^{\frac{1}{p-1}} m},
$$

where $z_{\lambda}=\lambda^{\frac{1}{p-1}} m a_{\lambda}$. Since $z_{\lambda} \rightarrow \infty$ as $\lambda \rightarrow \infty$,

$$
\liminf _{x \rightarrow \infty} \frac{f^{\frac{1}{p-1}}\left(c g^{\frac{1}{q-1}}(x)\right)}{x}=0 .
$$

This completes the proof of Theorem 3 .

\section{REFERENCES}

[1] H. Brezis and S. Kamin, Sublinear elliptic equations in $R^{n}$, Manuscripta Math. 74 (1992), 87-106. MR93f:35062

[2] R. Dalmasso, Existence and uniqueness of positive solutions of semilinear elliptic systems, Nonlinear Anal. 39 (2000), 559-568. MR2001d:35062

[3] D. Gilbarg and N. S. Trudinger, Elliptic partial differential equations of second order, Springer, Berlin, 1977. MR 57:13109

[4] D. D. Hai and R. Shivaji, An existence result on positive solutions for a class of semilinear elliptic systems, Proc. Roy. Soc. Edinburgh 134 A (2004), 137-141.

[5] G. M. Liberman, Boundary regularity for solutions of degenerate elliptic equations, Nonlinear Anal. 12 (1988), 1203-1219. MR90a:35098

[6] M. A. Krasnoselskii, Positive solutions of operator equations, Noordhoff, Groningen, 1964. MR31:6107

[7] J. l. Vazquez, A strong maximum principle for some quasilinear elliptic equations, Appl. Math. Optim. 12 (1984), 191-202. MR:86m:35018

Department of Mathematics, Mississippi State University, Mississippi State, MissisSIPPI 39762

E-mail address: dangera.msstate.edu 